\title{
Spontaneous Coronary Artery Dissection in a Postpartum Female: Case Report and Simplified Algorithm for its Diagnosis and Management
}

\author{
Nikhil Agrawal ${ }^{1}$, Arjun Khunger ${ }^{2}$, Kinan Dalal $^{3}$, Usman Zahid ${ }^{1}$, Timothy Petrie $^{3}$ \\ 1. Internal Medicine, University at Buffalo, Buffalo, USA 2. Oncology, Cleveland Clinic, Cleveland, USA 3. Cardiology, \\ University at Buffalo, Buffalo, USA
}

Corresponding author: Nikhil Agrawal, nikhilag@buffalo.edu

\begin{abstract}
Spontaneous coronary artery dissection is a rare, life-threatening cause of acute myocardial infarction that must always be considered in the differential diagnosis, particularly in young, healthy women with a paucity of typical risk factors for heart disease. We present a case of a 39-year-old White woman, three months postpartum, presenting with severe epigastric chest pain radiating to her neck. Subsequent workup using coronary angiography revealed spontaneous dissection of the distal left anterior descending artery. The patient was successfully managed by conservative treatment using low dose aspirin, metoprolol, and captopril, highlighting that hemodynamically stable patients with lesions in distal branches of coronary arteries and single-vessel disease can be successfully managed in a conservative fashion, without the need for surgical or percutaneous revascularization.
\end{abstract}

Received 03/18/2019

Review began 04/01/2019 Review ended 04/03/2019 Published 04/04/2019

(c) Copyright 2019

Agrawal et al. This is an open access article distributed under the terms of the Creative Commons Attribution License CC-BY 3.0., which permits unrestricted use, distribution, and reproduction in any medium, provided the original author and source are credited.
Categories: Cardiology, Emergency Medicine, Internal Medicine

Keywords: spontaneous coronary artery dissection, postpartum, pregnancy, interventional cardiology, angiography, ekg, transthoracic echocardiography, pci, cabg, medical treatment

\section{Introduction}

Spontaneous coronary artery dissection (SCAD) is a dissection of the coronary artery that is not related to trauma, iatrogenic causes, or atherosclerosis [1]. It is a highly unusual cause of acute coronary syndrome with a reported prevalence of $0.07 \%$ to $1 \%$ in the general population [2-4]. SCAD is most often associated with acute myocardial infarction (AMI) in women during their third trimester of pregnancy or the early postpartum period. Coronary dissection is thought to develop from either an intimal tear or a spontaneous hemorrhage in the vessel wall, forming a hematoma underneath the arterial wall and compressing the true coronary lumen leading to AMI. Early recognition and treatment is critical, given the high mortality rate associated with SCAD. We present the case of a 39-year-old female who presented to the hospital with chest pain and was found to have SCAD of the left anterior descending (LAD) artery on coronary angiography.

\section{Case Presentation}

A 39-year-old White female presented to the hospital with a three-day history of epigastric pain. She described her pain as a burning pain that started at rest and was radiating to her neck. Her pain was associated with shortness of breath, and she denied any episodes of nausea, vomiting, cough, fevers, chills, or sweating. She recently had a twin pregnancy and gave birth at about 32 weeks of gestation via caesarian section, but lost both her children after birth. This event had been very emotionally taxing on her for the past three months.

Her past medical history was only remarkable for gestational diabetes during her last pregnancy. She typically smoked one pack per day of cigarettes for the last 20 years. Recently, she increased her smoking to two packs per day for the past few months. She denied any use of alcohol or recreational drugs. She also denied any other cardiac risk factors, significant family history of cardiac disease, sudden cardiac death, or connective tissue disease. She had no allergies and was not taking any medications.

On examination, she was afebrile with a temperature of $36.9^{\circ} \mathrm{C}$, heart rate of 82 beats $/ \mathrm{min}$, a blood pressure of $165 / 110$, respiratory rate of 18 breaths/minute, and oxygen saturation of $99 \%$ on room air. Her head and neck examination were unremarkable with no jugular venous distention. On auscultation, there was good air entry bilaterally and normal heart sounds with no murmurs, rubs, or gallops. There was no edema on examination of the lower extremities.

An initial electrocardiogram (EKG) was obtained as can be seen in Figure 1. 


\section{Cureus}

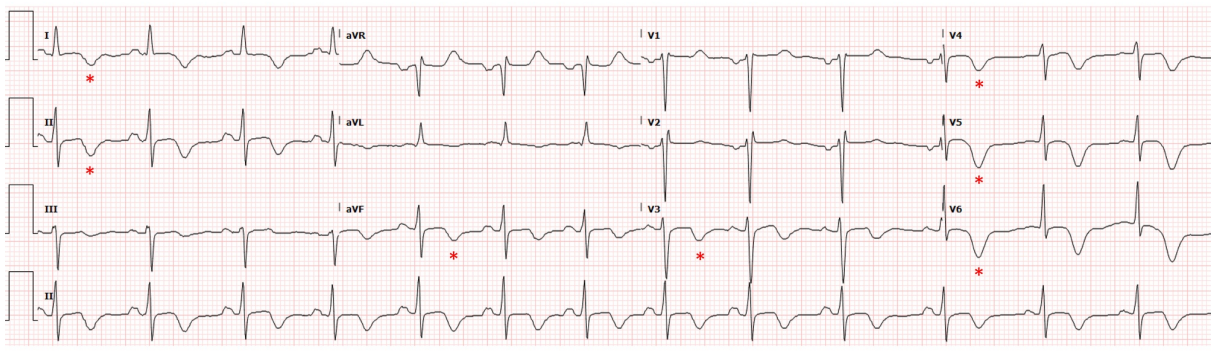

FIGURE 1: Electrocardiogram (EKG) on presentation showing sinus rhythm with sinus arrhythmia and $T$ wave inversions concerning for anterior ischemia (asterisks).

Her EKG showed sinus rhythm with sinus arrhythmia and anterolateral $\mathrm{T}$ wave inversions concerning for anterior ischemia. Cardiac biomarkers included a creatine kinase level of $261 \mathrm{U} / \mathrm{L}$ (normal range, 33-211 $\mathrm{U} / \mathrm{L}$ ), a creatine kinase-MB fraction of $13 \mathrm{U} / \mathrm{L}$ (normal, $<10 \mathrm{U} / \mathrm{L}$ ), and a troponin I level of $5.22 \mathrm{ng} / \mathrm{mL}$ (normal, $<0.01 \mathrm{ng} / \mathrm{mL}$ ). She was transferred from an urgent care facility after aspirin and ticagrelor loading for percutaneous coronary intervention. Her peak troponin I was 5.4. Angiography revealed spontaneous coronary artery dissection (SCAD) of the distal left anterior descending (LAD) artery without any other atherosclerotic disease (Figures 2-3). The other coronary arteries were normal. Catheterization also revealed a mildly reduced left ventricular ejection fraction of $45 \%$ and akinetic apical segments of the left ventricle.
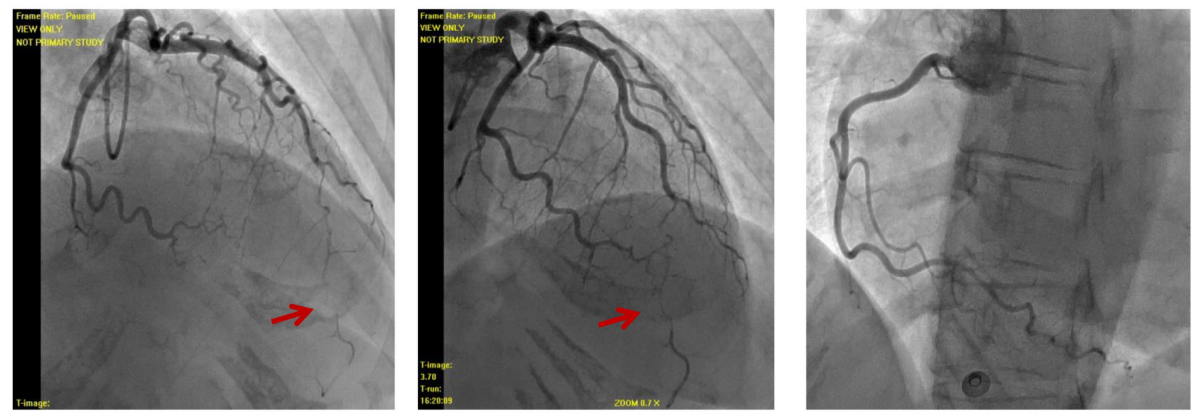

FIGURE 2: Angiography of the left coronary artery (left and middle image) and right coronary artery (right image). Left coronary angiography showing spontaneous coronary artery dissection of the distal left anterior descending artery with TIMI 2 flow (arrows).

TIMI - Thrombolysis in Myocardial Infarction
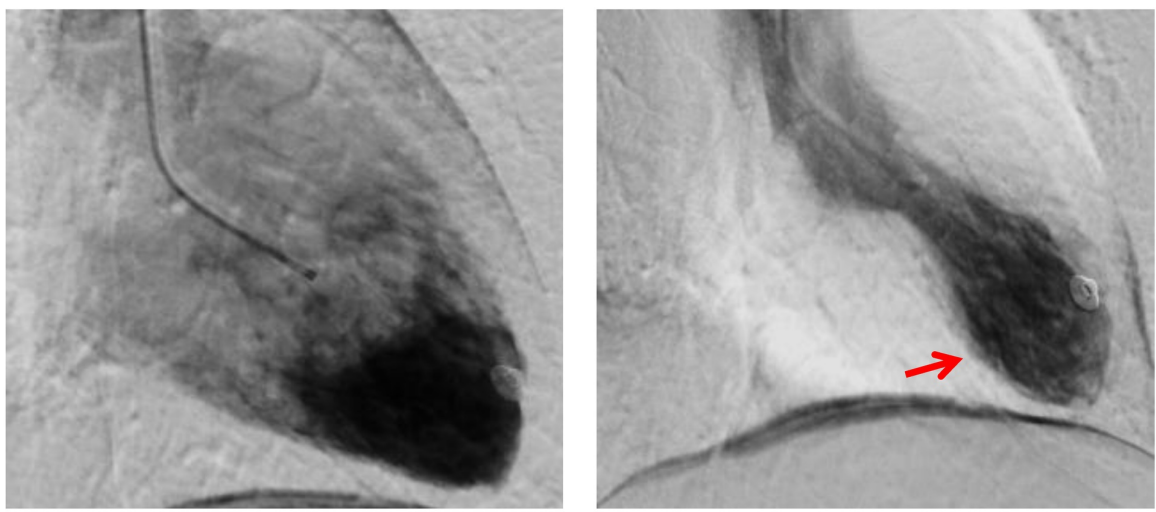

FIGURE 3: Angiography revealed akinetic apical segments (arrow) with 
She was started on medical management for SCAD, which included low dose aspirin, metoprolol, and captopril. Dual antiplatelet therapy and heparin were not given because of the risk for increased bleeding and no clear evidence of benefit. She was admitted to the cardiac critical care unit for observation and remained hemodynamically stable during her stay. Echocardiography done after one day confirmed wall motion abnormalities of the left ventricular apex with an estimated low normal left ventricular ejection fraction of 50\%-55\% (Figure 4). Laboratory workup for possible connective tissue disorders or vasculitis was done as underlying coronary vasculitis may increase the risk for spontaneous dissection and it was negative for rheumatoid factor, anti-CCP and ANA and only positive for atypical perinuclear anti-neutrophil cytoplasmic antibodies (P-ANCA). She was discharged two days after the presentation on medical therapy. The patient was doing well on the subsequent follow-up visit.
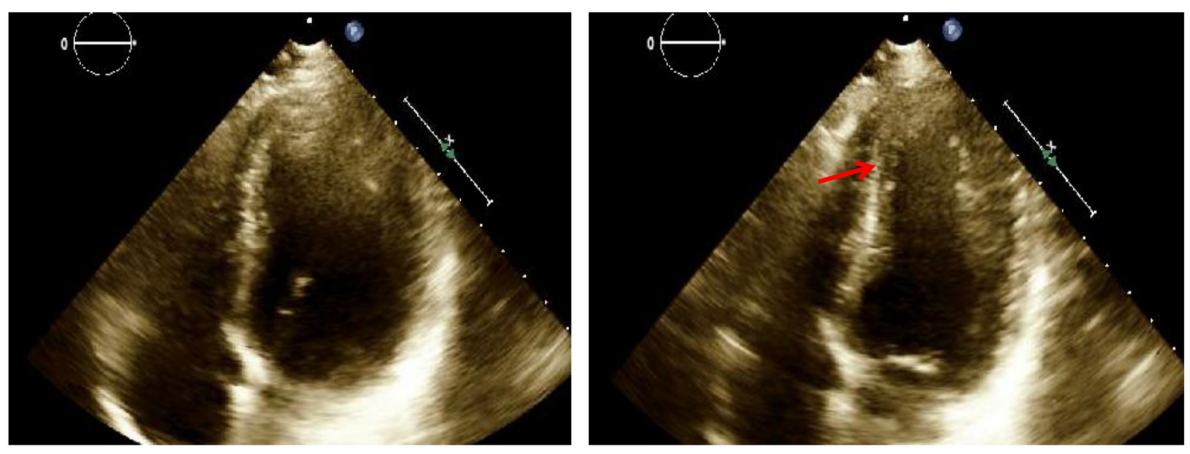

FIGURE 4: Transthoracic echocardiography on day 2 revealed regional wall motion abnormalities of the left ventricular apex with a low normal estimated left ventricular ejection fraction of $50 \%-55 \%$. The right ventricle had normal systolic function and size. The left ventricle in four chamber view is shown in diastole (left) and systole (right) with an akinetic apex (arrow). No left ventricular thrombus was present.

\section{Discussion}

Spontaneous coronary artery dissection in the postpartum period is a rare but life-threatening event and several cases have been reported in the past [5-11]. The pathogenesis of pregnancy related-SCAD remains uncertain, and it is suggested that hormonal and hemodynamic changes during the peripartum period weaken the coronary artery walls, predisposing them to acute dissection. Women of advanced age are reported to have a higher risk of myocardial infarction (MI). Other risk factors for SCAD include systemic arteriopathies such as fibromuscular dysplasia, connective tissue disorders, extreme emotional stress, family history of SCAD, and, less frequently, coronary vasospasm and cocaine abuse [12,13].

Pregnancy-related SCAD is reported to occur up to three months after delivery in almost $80 \%$ of cases, at a median time of 13 days postpartum [14]. Dissection may be seen in any coronary artery; however, the LAD or left circumflex is involved in about $80 \%$ of cases [6]. The dissections may be multifocal in about $40 \%$ of the cases [6]. In this case, the combination of hormonal changes during pregnancy, advanced maternal age, history of severe emotional distress and smoking is the most likely etiology for this patient's MI secondary to coronary artery dissection.

Clinically, SCAD can present as stable or unstable angina, myocardial infarction, congestive heart failure, cardiac tamponade, cardiogenic shock, or sudden cardiac death $[15,16]$. As in the non-pregnant population, chest pain is the most common symptom at the initial presentation. Other common symptoms include nausea, diaphoresis, and breathlessness [12]. Initial evaluation includes an electrocardiogram, cardiac biomarkers, and a transthoracic echocardiogram. The diagnosis of SCAD is typically confirmed with the use of conventional coronary angiography. Figure 5 presents an algorithm for the diagnosis and management of SCAD in a postpartum female. 


\section{Cureus}

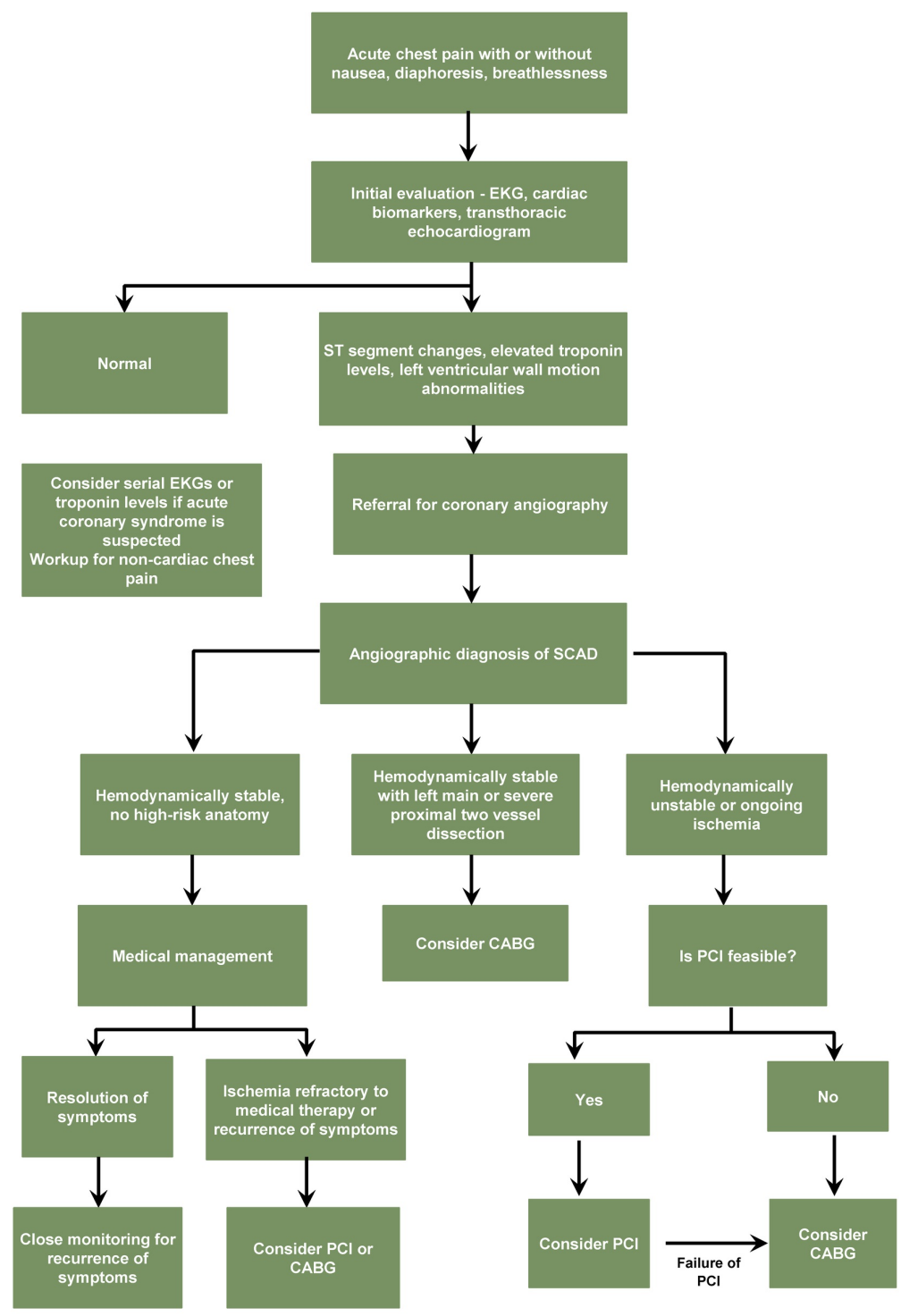

FIGURE 5: Algorithm for the diagnosis and management of spontaneous coronary artery dissection (SCAD) in a postpartum female

CABG: Coronary Artery Bypass Graft; EKG: Electrocardiogram; PCI: Percutaneous Coronary Intervention; SCAD: Spontaneous Coronary Artery Dissection

Management of SCAD should be individualized, based on the patient's hemodynamic status, the site of dissection, and the extent of vessel involvement and may range from conservative medical therapy to percutaneous coronary intervention (PCI) or coronary artery bypass graft (CABG). For hemodynamically stable patients with lesions in distal branches of coronary arteries and single-vessel disease, medical treatment with antiplatelets (acetylsalicylic acid, clopidogrel), glycoprotein IIb/IIIa inhibitors, beta blockers, and nitroglycerin may be the best choice [17]. In patients with ongoing ischemia, unstable hemodynamics, or left main artery involvement, PCI or CABG should be considered [1]. PCI in SCAD is associated with complications such as extending the dissection and guidewires entering the false lumen [1]. In patients with unstable hemodynamics, multi-vessel involvement, failure of attempted PCI, or left main stem or proximal artery dissection, CABG is typically the treatment of choice [1]. Most of the studies discourage the use of fibrinolytic agents due to the risk of extension of the coronary dissection and worsening of any coronary spasm by compressing the vascular lumen [18]. Given the location of the dissection (distal LAD) and stable hemodynamic status of our patient, we chose to proceed with conservative therapy with low dose aspirin, metoprolol, and captopril. Further, recurrence of SCAD may be seen in $5.2 \%$ to $17 \%$ of patients, and they should be closely followed for any recurrent events or cardiac complications.

\section{Conclusions}

Spontaneous coronary artery dissection (SCAD) should be strongly considered in the differential diagnosis 
for young women who present with signs and symptoms of acute coronary syndrome during pregnancy or the postpartum period. PCI has an increased risk of complications, and recurrence rates are high. Management requires a multidisciplinary approach and depends upon the clinical presentation, coronary anatomy, and hemodynamic stability of the patient.

\section{Additional Information \\ Disclosures}

Human subjects: Consent was obtained by all participants in this study. Conflicts of interest: In compliance with the ICMJE uniform disclosure form, all authors declare the following: Payment/services info: All authors have declared that no financial support was received from any organization for the submitted work. Financial relationships: All authors have declared that they have no financial relationships at present or within the previous three years with any organizations that might have an interest in the submitted work. Other relationships: All authors have declared that there are no other relationships or activities that could appear to have influenced the submitted work.

\section{References}

1. Hayes SN, Kim ES, Saw J, et al.: Spontaneous coronary artery dissection: current state of the science: a scientific statement from the American Heart Association. Circulation. 2018, 137:523-557. 10.1161/CIR.0000000000000564

2. Vanzetto G, Berger-Coz E, Barone-Rochette G, et al.: Prevalence, therapeutic management and mediumterm prognosis of spontaneous coronary artery dissection: results from a database of 11,605 patients. Eur J Cardiothorac Surg. 2009, 35:250-254. 10.1016/j.ejcts.2008.10.023

3. Mortensen KH, Thuesen L, Kristensen I, Christiansen EH: Spontaneous coronary artery dissection: a Western Denmark Heart Registry study. Catheter Cardiovasc Interv. 2009, 74:710-717. 10.1002/ccd.22115

4. Nishiguchi T, Tanaka A, Ozaki Y, et al.: Prevalence of spontaneous coronary artery dissection in patients with acute coronary syndrome. Eur Heart J Acute Cardiovasc Care. 2016, 5:263-270. $10.1177 / 2048872613504310$

5. Køber HE, Lars: Spontaneous coronary artery dissection: case report and literature review . Scand Cardiovasc J. 1999, 33:175-179. 10.1080/14017439950141821

6. Koul AK, Hollander G, Moskovits N, Frankel R, Herrera L, Shani J: Coronary artery dissection during pregnancy and the postpartum period: two case reports and review of literature. Catheter Cardiovasc Interv. 2001, 52:88-94. 10.1002/1522-726X(200101)52:1<88::AID-CCD1022>3.0.CO;2-P

7. Higgins GL, Borofsky JS, Irish CB, Cochran TS, Strout TD: Spontaneous peripartum coronary artery dissection presentation and outcome. J Am Board Fam Med. 2013, 26:82-89. 10.3122/jabfm.2013.01.120019

8. Okamoto M, Tanaka M, Ishii M, et al.: A case of postpartum spontaneous coronary artery dissection . Gen Thorac Cardiovasc Surg. 2014, 62:726-729. 10.1007/s11748-013-0274-9

9. Pulivarthi S, Lawal T, Li D, Gurram MK: Spontaneous coronary artery dissection and acute myocardial infarction after cesarean section in a postpartum woman with untreated dyslipidemia. Heart Views. 2014, 15:80. 10.4103/1995-705X.144795

10. Moussa HN, Movahedian M, Leon MG, Sibai BM: Acute myocardial infarction due to coronary artery dissection in the postpartum period. AJP reports. 2015, 5:093. 10.1055/s-0035-1547330

11. Aprigliano G, Palloshi A, Morici N: Spontaneous left main stem coronary artery dissection in a young postpartum woman. J Cardiovasc Med. 2017, 18:721-722. 10.2459/JCM.0b013e32835ec7ca

12. Tweet MS, Hayes SN, Pitta SR, et al.: Clinical features, management and prognosis of spontaneous coronary artery dissection. Circulation. 2012, 126:579-588. 10.1161/CIRCULATIONAHA.112.105718

13. Hayes SN: Spontaneous coronary artery dissection (SCAD): new insights into this not-so-rare condition . Tex Heart Inst J. 2014, 41:295-298. 10.14503/THIJ-14-4089

14. Maeder M, Ammann P, Drack G, Rickli H: Pregnancy-associated spontaneous coronary artery dissection: impact of medical treatment. Zeitschrift für Kardiologie. 2005, 94:829-835. 10.1007/s00392-005-0302-6

15. Saw J, Aymong E, Sedlak T, et al.: Spontaneous coronary artery dissection: association with predisposing arteriopathies and precipitating stressors and cardiovascular outcomes. Circ Cardiovasc Interv. 2014, 7:645655. 10.1161/CIRCINTERVENTIONS.114.001760

16. Havakuk O, Goland S, Mehra A, Elkayam U: Pregnancy and the risk of spontaneous coronary artery dissection: an analysis of 120 contemporary cases. Circ Cardiovasc Interv. 2017, 10:004941. 10.1161/CIRCINTERVENTIONS.117.004941

17. Tweet MS, Eleid MF, Best PJ, et al.: Spontaneous coronary artery dissection: revascularization versus conservative therapy. Circ Cardiovasc Interv. 2014, 7:777-786. 10.1161/CIRCINTERVENTIONS.114.001659

18. Shahzad K, Cao L, Ain QT, Waddy J, Khan N, Nekkanti R: Postpartum spontaneous dissection of the first obtuse marginal branch of the left circumflex coronary artery causing acute coronary syndrome: a case report and literature review. J Med Case Rep. 2013, 7:82. 10.1186/1752-1947-7-82 